\title{
Tailored Polymer Particles with Ordered Network Structures in Emulsion Droplets
}

Xiaolin Lyu, ${ }^{\dagger}$, Zhehao Tang, ${ }^{\dagger}$ Yujie Li,,${ }^{\S}$ Anqi Xiao,${ }^{\dagger}$ Zhihao Shen, ${ }^{*, \dagger}$ Shijun Zheng, ${ }^{\S}$ Xing-He Fan ${ }^{\dagger}$

${ }^{\dagger}$ Beijing National Laboratory for Molecular Sciences, Key Laboratory of Polymer Chemistry and Physics of Ministry of Education, Center for Soft Matter Science and Engineering, College of Chemistry and Molecular Engineering, Peking University, Beijing 100871, China

Key Laboratory of Advanced Materials Technologies, College of Materials Science and Engineering, Fuzhou University, Fuzhou 350108, China

${ }^{\S}$ College of Materials Science and Engineering, Zhengzhou University, Zhengzhou 450000, China

E-mail: zshen@pku.edu.cn

\section{Table of Contents}

Table S1. Molecular characteristics of the macroinitiator and DM $\quad$ S2

Scheme S1. Synthetic procedure of PDMS- $b$-PMPCS. $\quad$ S2

Figure S1. GPC and ${ }^{1} \mathrm{H}$ NMR results of the macroinitiator and DM $\quad$ S2

Figure S2. TGA and DSC results of the DM S3

Figure S3. TEM micrographs of polymer particles prepared from chloroform emulsion $\quad$ S3 droplets

Figure S4. SEM and TEM micrographs of polymer particles prepared from dichloromethane emulsion droplets.

Figure S5. SEM and TEM micrographs of polymer particles prepared from dichloroethane emulsion droplets. 
Table S1. Molecular characteristics of PDMS-Br and DM

\begin{tabular}{|c|c|c|c|}
\hline Notation & $\boldsymbol{M}_{\mathbf{n}}(\mathbf{g} / \mathbf{m o l}){ }^{\boldsymbol{a}}$ & $\boldsymbol{\oplus}_{\mathbf{M}}{ }^{\boldsymbol{b}}$ & $\boldsymbol{f}_{\text {PMPCS }}(\boldsymbol{\%})^{\boldsymbol{c}}$ \\
\hline PDMS-Br & 4900 & 1.17 & \\
\hline DM & 16100 & 1.18 & 64 \\
\hline
\end{tabular}

${ }^{a}$ The polymerization degree of PMPCS and number-averaged molecular weight were determined by ${ }^{1} \mathrm{H}$ NMR.

${ }^{b}$ The molar-mass dispersity $\left(\bigoplus_{\mathrm{M}}\right)$ were determined by GPC.

${ }^{c}$ The densities of PDMS and PMPCS are $0.97 \mathrm{~g} / \mathrm{cm}^{3}$ and $1.28 \mathrm{~g} / \mathrm{cm}^{3}$, respectively.

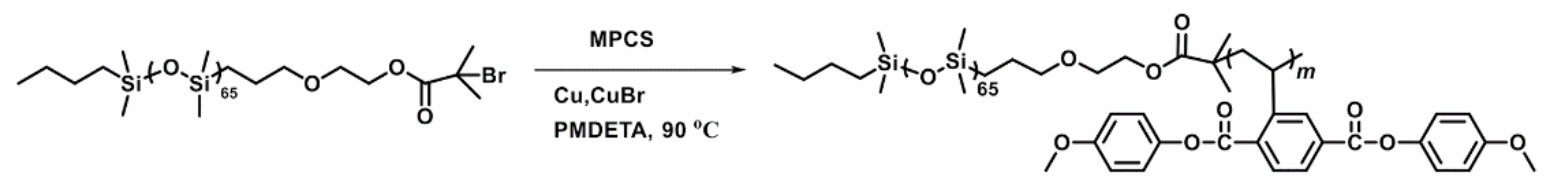

Scheme S1. Synthetic procedure of PDMS- $b$-PMPCS.

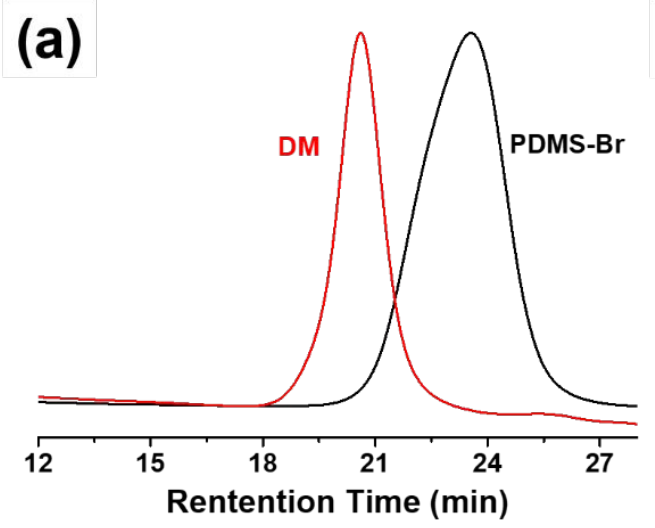

(b)

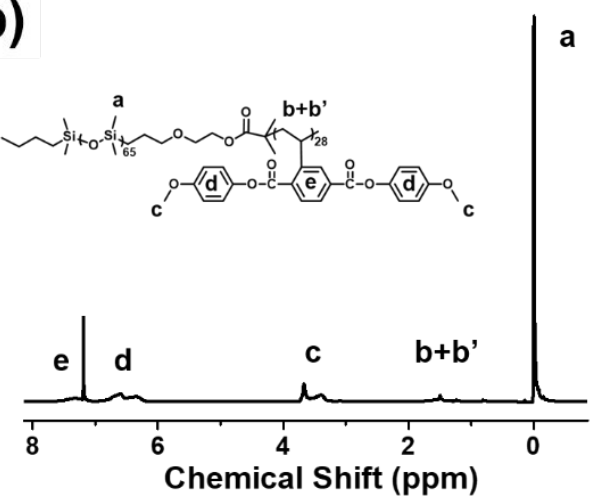

Figure S1. GPC curves of DM and PDMS-Br (a) and ${ }^{1} \mathrm{H}$ NMR spectrum of DM with $\mathrm{CDCl}_{3}$ as the solvent (b). 

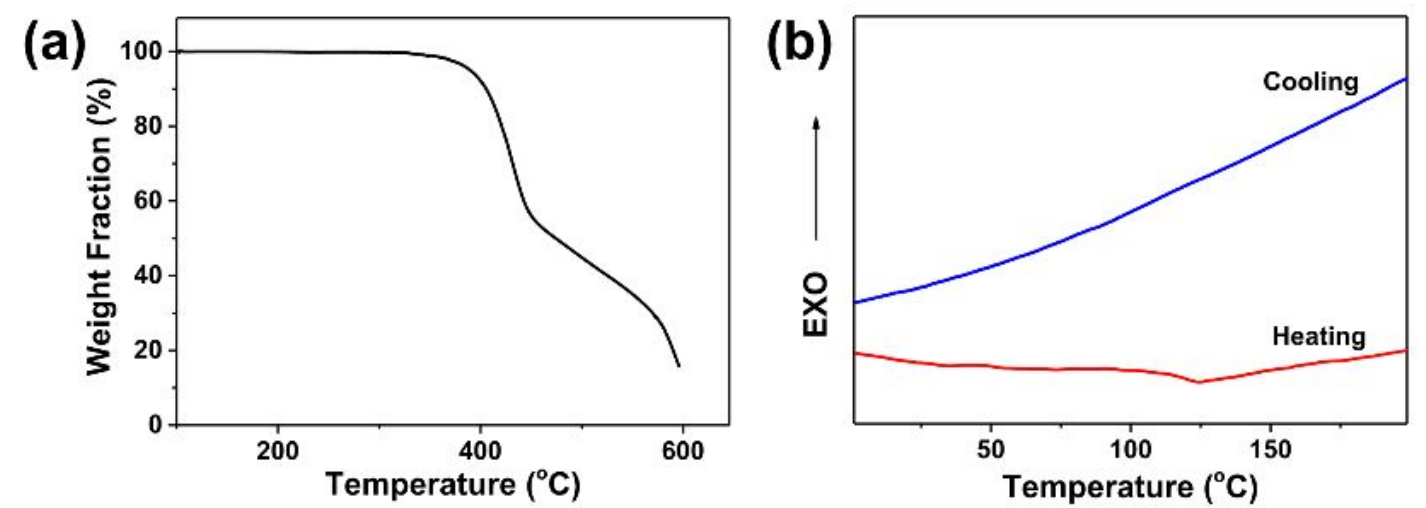

Figure S2. TGA curve of DM at a scanning rate of $10^{\circ} \mathrm{C} / \mathrm{min}$ under a nitrogen atmosphere (a) and DSC thermograms of $\mathrm{DM}$ at a heating rate of $20^{\circ} \mathrm{C} / \mathrm{min}$ and a cooling rate of $5{ }^{\circ} \mathrm{C} / \mathrm{min}$ under a nitrogen atmosphere (b).
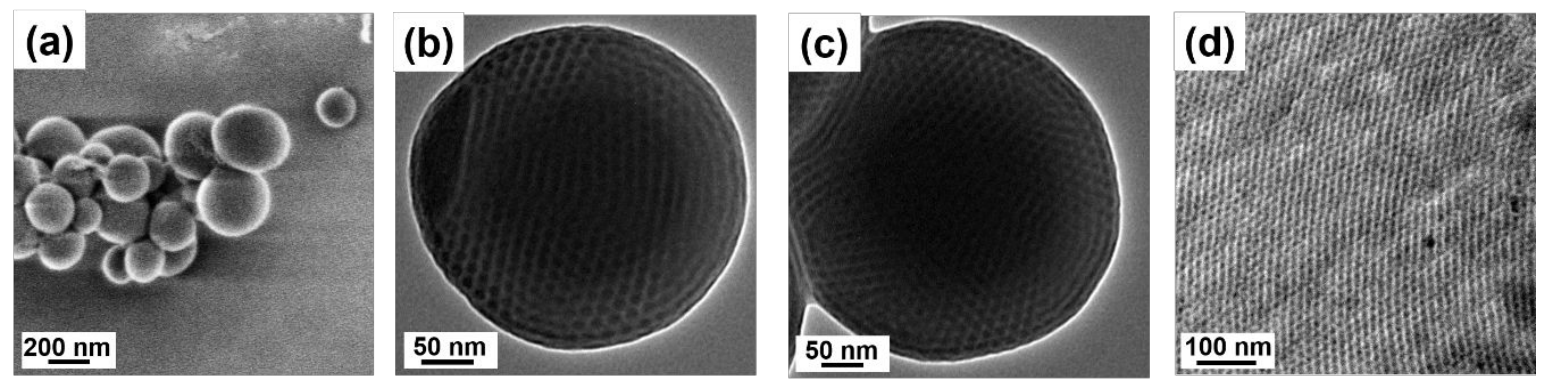

Figure S3. SEM (a) and TEM (b, c) micrographs of polymer particles prepared from chloroform emulsion droplets and thin slice of the particles after being sectioned by ultramicrotomy (d).
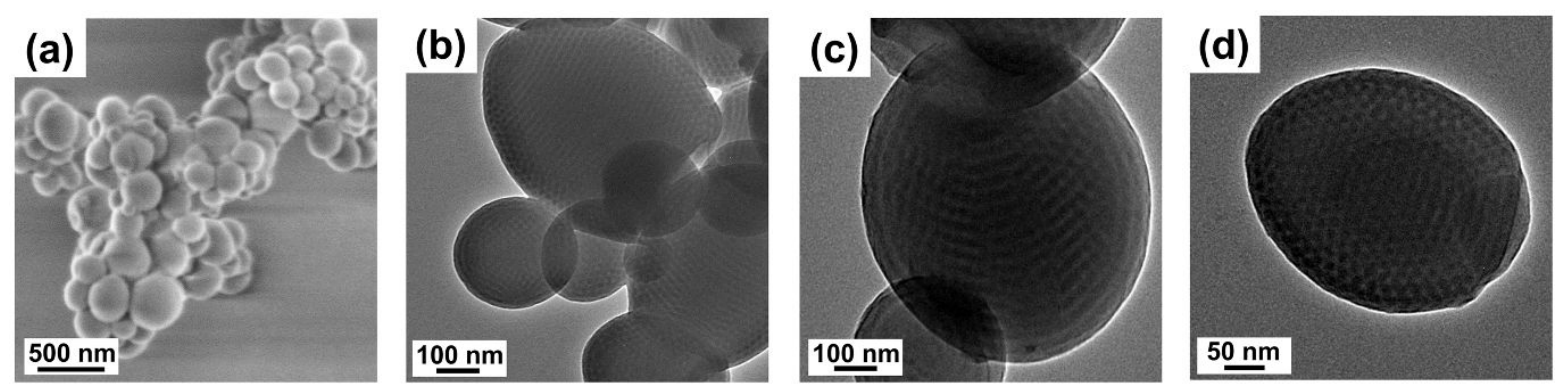

Figure S4. SEM (a) and TEM (b, c, d) micrographs of polymer particles prepared from dichloromethane emulsion droplets. 

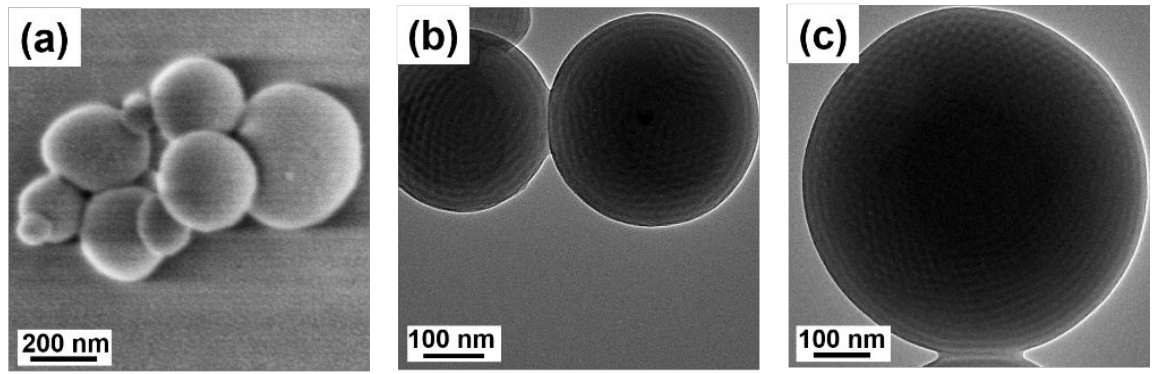

Figure S5. SEM (a) and TEM (b, c) micrographs of polymer particles prepared from dichloroethane emulsion droplets. 